tology Department, we retrospectively selected 35 patients that within one year from the first visit had a defined diagnosis of RA (according with the 2010 ACR/EULAR criteria) or of axial/peripheral SpA (according with the 2009 ASAS criteria). Demographic, clinical parameters and MCPs' MSUS assessment at baseline were recorded during the patients' first EA clinic visit by an experienced Rheumatologist and by a blinded skilled sonographer. ESAOTE MyLAB 70 with $6-18 \mathrm{MHz}$ linear array transducer was used for all patients scanning and US were scored according with OMERACT guidelines.

Results: MSUS data of twenty RA patients $(17 \mathrm{~F}-3 \mathrm{M}$; median age of 59 yrs with range 35-83 yrs, median time for defined diagnosis of 2,4 months with range $1-11$ months) and $15 \mathrm{SpA}$ patients (9F-6M; median age of 53 yrs with range 18-78 yrs, median time for defined diagnosis of 1,7 months with range 1-8 months) were retrospectively analyzed. At the first EA clinic visit, all patients showed at least one joint with synovial fluid and/or synovial hypertrophy in Grey scale (GS) with a score $>1$ and no statistically significant differences were found in the percentage of patients that presented at least one joint with power Doppler (PD) positivity (55\% RA Vs $53 \%$ SpA, $p=0,92)$ and that presented PD positivity tenosynovitis of the flexor tendons in at least one finger (10\% RA Vs $33 \%$ $\mathrm{SpA}, \mathrm{p}=0,08)$. A statistically significant difference was found in the percentage of patients with erosions in at least one MCP $(25 \%$ RA Vs $0 \% \mathrm{SpA}, \mathrm{p}=0,036)$ and in the percentage of patients with PD positive paratenonitis of the extensor tendons in at least one finger ( $30 \%$ RA Vs $80 \%$ SpA pts, $p=0,003)$.

Conclusions: Early RA patients showed a statistically higher percentage of erosions at MCPs' MSUS evaluation of the first visit of the EA clinic, when compared with early SpA group and early SpA patients presented at the level of the extensor tendons an higher percentage of PD positive paratenonitis when compared with the early RA group.

References:

[1] Lage-Hansen PR. et al. The role of ultrasound in diagnosing rheumatoid arthritis, what do we know? An updated review. Rheumatol Int.2017Feb;37(2):179187.

[2] Gutierrez M. et al. Differential diagnosis between rheumatoid arthritis and psoriatic arthritis: the value of ultrasound findings at metacarpophalangeal joints level.Ann Rheum Dis.2011 Jun;70.

Disclosure of Interest: None declared

DOI: 10.1136/annrheumdis-2017-eular.5658

\section{SAT0621 AGREEMENT BETWEEN SEMIQUANTITATIVE AND QUANTITATIVE DOPPLER SCORING SYSTEMS FOR THE ASSESSMENT OF SYNOVIAL PATHOLOGICAL VASCULARIZATION IN RHEUMATOID ARTHRITIS}

E. Naredo ${ }^{1,2}$, I.M. Rutigliano ${ }^{3}$, I. Janta ${ }^{1}$, C. Scirocco ${ }^{3}$, L. Valor ${ }^{1}$, F. Figus ${ }^{4}$, L. Carreño ${ }^{1}$, J. Garrido ${ }^{5}$, A. lagnocco ${ }^{6} .{ }^{1}$ Department of Rheumatology, Hospital General Universitario Gregorio Marañón: ${ }^{2}$ Department of Rheumatology, Hospital Universitario Fundación Jiménez Díaz. Autonoma University, Madrid, Spain; ${ }^{3}$ Dipartimento Medicina Interna e Specialità Mediche, Sapienza Università di Roma, Rome; ${ }^{4}$ Dipartimento Reumatologia, Università degli Studi di Cagliari, Cagliari, Italy; ${ }^{5}$ Department of Social Psychology and Methodology, Faculty of Psychology, Autonoma University of Madrid, Madrid, Spain: ${ }^{6}$ Dipartimento Scienze Cliniche e Biologiche, Università degli Studi di Torino, Turin, Italy

Objectives: To compare power Doppler (PD) vs colour Doppler (CD) semiquantitative and quantitative scoring of synovial vascularization (RA) and to evaluate the relationship between semiquantitative and quantitative scores in patients with rheumatoid arthritis (RA).

Methods: One hundred RA patients underwent B-mode, PD, and CD assessments of 12 joints at two European centres. Each joint with synovial hypertrophy detected on B-mode was semiquantitatively scored (0-3) for PD (SPD score) and CD (SCD score) synovial signal. $P D$ and $C D$ synovial signal were also quantitatively scored $(0-100 \%)$ (QPD and QCD scores, respectively) using a software for counting the colour fraction.

Results: We found SH in 184 joints. SPD and SCD agreed in $92.3 \%$ (95\% Cl: 88.4; $96.2 \%$ ) of paired scores, with Kendall rank correlation coefficient tau-b=0.95. Significant differences between marginal distributions of SPD and SCD were not found $(p=0.565)$. QPD and QCD scores were highly correlated (Pearson's coefficient=0.70) but Blamd-Altman plot showed insufficient agreement, being the QCD scores systematically slightly higher than the QPD scores. The distribution of QPD and QCD values between SPD and SCD scores, respectively, showed significant differences between grade 0 and grade $1(p<0.001)$, and grade 2 and grade 3 ( $p=0.042$ and $p=0.007$, respectively) but not between grade 1 and 2 $(\mathrm{p}=0.154$ and $\mathrm{p}=0.150$, respectively).

Conclusions: SPD and SCD scores were concordant and QPD and QCD scores highly correlated although were not concordant. There was consistency between SPD and SCD moderate and severe scores and QPD and QCD scores. There was an overlapping between SPD and SCD mild and moderate scores regarding QPD and QCD scores.

Disclosure of Interest: None declared

DOI: 10.1136/annrheumdis-2017-eular.3251

\section{SAT0622 MULTICENTER 14-3-3ETA BIOMARKER REPRODUCIBILITY; THE JAPANESE EXPERIENCE}

S. Hirata ${ }^{1,2}$, M. Kaneda ${ }^{3}$, T. Isayama ${ }^{3}$, Y. Gui ${ }^{4}$, A. Marotta ${ }^{4}$,

W.P. Maksymowych ${ }^{5}$, Y. Tanaka ${ }^{2}{ }^{1}$ Hiroshima University Hospital, Hiroshima; ${ }^{2}$ University of Occupational and Environmental Health, Kitakyushu; ${ }^{3}$ Medical \& Biological Laboratories, Nagano-ken, Japan; ${ }^{4}$ Augurex Life Sciences Corp, Vancouver; ${ }^{5}$ University of Alberta, Edmonton, Canada

Background: The soluble biomarker sub-committee of OMERACT has published validation criteria for biomarkers reflecting structural damage end-points. Within these, categories of Discrimination and Feasibility relate to assay reproducibility and performance. 14-3-3eta $(\eta)$ is a joint-derived biomarker that drives joint damage processes and informs radiographic progression independently of acute phase reactants. As an ELISA assay, 14-3-3n is currently available for clinical use as a laboratory developed test (LDT) in the United States (US) and as an in-vitro diagnostic (IVD) in Canada. The ELISA is (CE) marked for Europe and is Therapeutics Good Administration (TGA) approved for Australia. Previous studies have described 14-3-3eta assay equivalence at independent laboratories in the US and Canada.

Objectives: The purpose of this study was to demonstrate the reproducibility of 14-3-3eta measurements at two independent laboratories in Japan and one in Canada.

Methods: A total of 212 samples from the University of Occupational and Environmental Health were provided for this study. Serum 14-3-3eta measurements were performed using the 14-3-3eta ELISA provided by Augurex. Testing in Canada occurred in August 2015; these 14-3-3eta values were set as the "standard". The 212 samples were shipped from Canada to Japan in January 2016 with testing being performed in February 2016. Testing at MBL was performed on a blinded-basis. Upon completion of testing, results were sent to the investigators at which point in time, MBL was unblinded. 14-3-3eta positivity was defined at $\geq 0.19 \mathrm{ng} / \mathrm{ml}$. Contigency and Spearman analyses were performed to assess the strength of the results between the two testing centres. Values above the linear range of the assay i.e. $\geq 20 \mathrm{ng} / \mathrm{ml}$ were excluded for the Spearman analyses and determination of the median (IQR). A p-value of $<0.05$ established statistical significance.

Results: In Canada, of the 212 samples tested, 146 (68.8\%) were 14-3-3eta positive and in Japan, $147(69.3 \%)$ were positive. In Canada, 187 patients had reportable values in the linear range and in Japan 186 did. Median 14-3-3eta levels in Canada were $0.51 \mathrm{ng} / \mathrm{ml}$ (IQR: 0.11-2.09) and in Japan they were 0.58 $\mathrm{ng} / \mathrm{ml}$ (IQR: $0.02-2.68$ ), respectively. Spearman correlation analysis revealed a highly significant correlation between the two testing sites, $r=0.92 ; p<0.00001$. Contingency analysis revealed a strong concordance between the two sets of results delivering a likelihood ratio $(L R)$ of $156, p<0.0001$. Between the two sites, 58 of the samples were 14-3-3eta negative and 139 were positive; there was agreement in $197(93 \%)$ of the 212 samples.

Conclusions: As defined by the soluble biomarker sub-committee of OMERACT, reliability and standardization of biomarker assessments in routine clinical practice across the globe and in clinical studies is of high importance. The results presented herein demonstrate that the 14-3-3eta assay is highly reliable, that testing/delivery of results can be standardized between two independent laboratories, and that it is very stable over time and when shipped between continents.

Disclosure of Interest: S. Hirata: None declared, M. Kaneda Employee of: Medical \& Biological Laboratories, T. Isayama Employee of: Medical \& Biological Laboratories, Y. Gui Employee of: Augurex Life Sciences Corp., A. Marotta Employee of: Augurex Life Sciences Corp., W. Maksymowych Consultant for: Augurex Life Sciences Corp, Y. Tanaka: None declared DOI: 10.1136/annrheumdis-2017-eular.5543

\section{SAT0623 CORRELATIONS BETWEEN CLINICAL, ULTRASOUND AND DISEASE ACTIVITY SCORES OF PERIPHERAL ENTHESITIS IN SPONDYLOARTHRITIS (SPA)}

A. Haddouche, S. Haid, S. Bencheikh, S. Slimani, A. Abdessemed, N. Brahimi, A. Ladjouze. Medicine, University of Algiers 2, Algiers, Algeria

Objectives: To look for correlations between clinical, ultrasound (US) and disease activity scores of peripheral enthesitis in an SpA cohort.

Methods: A prospective study of all SpAs meeting SpA ASAS criteria followed at EHS Ben Aknoun, over a period from January 2015 to April 2016. Seventeen entheseal sites were assessed bilaterally: insertions of supra-spinatus, infraspinatus, sub-scapular, medial and lateral epicondylars, triceps brachialis, gluteus midius and minimus, quadricipital, proximal and distal insertion (patellar ligament, medial and lateral collateral ligament), Achilles tendon and plantar aponeurosis. Peripheral entheses was assessed by the following clinical scores: Enthesitis Peripheral Score (PES = Sum of symptomatic peripheral entheses sites on clinical examination), Visual Analog Scale of peripheral enthesitis (VAS), Spondyloarthritis Research Consortium of Canada score (SPARCC) as well as the following US enthesitis scores: Acute Enthesitis score (Sum of acute enthesitis US scores for each site), Chronic Enthesitis score (Sum of US chronic enthesitis scores for each site), Global Enthesitis score (Sum of the acute and chronic US scores of enthesitis), Doppler signal Enthesitis score (Sum of Doppler signal scores less than $2 \mathrm{~mm}$ from cortical bone for each site), Madrid Sonography Enthesitis Index (MASEI), Simplified Echographic Score (SES) wich assesses only the Achilles 